\title{
Impact of catheter antimicrobial coating on species-specific risk of catheter colonization: a meta-analysis
}

\author{
Aleksey Novikov ${ }^{1}$, Manuel Y Lam¹, Leonard A Mermel ${ }^{2,3^{*}}$, Anna L Casey ${ }^{4}$, Tom S Elliott ${ }^{4}$ and Peter Nightingale ${ }^{5}$
}

\begin{abstract}
Background: Antimicrobial catheters have been utilized to reduce risk of catheter colonization and infection. We aimed to determine if there is a greater than expected risk of microorganism-specific colonization associated with the use of antimicrobial central venous catheters (CVCs).

Methods: We performed a meta-analysis of 21 randomized, controlled trials comparing the incidence of specific bacterial and fungal species colonizing antimicrobial CVCs and standard CVCs in hospitalized patients.

Results: The proportion of all colonized minocycline-rifampin CVCs found to harbor Candida species was greater than the proportion of all colonized standard CVCs found to have Candida. In comparison, the proportion of colonized chlorhexidine-silver sulfadiazine CVCs specifically colonized with Acinetobacter species or diphtheroids was less than the proportion of similarly colonized standard CVCs. No such differences were found with CVCs colonized with staphylococci.

Conclusion: Commercially-available antimicrobial CVCs in clinical use may become colonized with distinct microbial flora probably related to their antimicrobial spectrum of activity. Some of these antimicrobial CVCs may therefore have limited additional benefit or more obvious advantages compared to standard CVCs for specific microbial pathogens. The choice of an antimicrobial CVC may be influenced by a number of clinical factors, including a previous history of colonization or infection with Acinetobacter, diphtheroids, or Candida species.
\end{abstract}

Keywords: Central venous catheter, Catheter colonization, Catheter-related bloodstream infection, Central line-associated bloodstream infection, Bacteremia, Antimicrobial catheter

\section{Introduction}

Central venous catheters (CVCs) have become essential in the management of critically ill patients, as well as other patient populations requiring acute or long-term medical care. Intravascular catheters can become colonized by microbial pathogens following an extraluminal or intraluminal route of endemic infection emanating from the insertion site and catheter connector/hub, respectively [1]. Meta-analyses have been published demonstrating a reduced risk of $\mathrm{CVC}$ colonization and CVC-related bloodstream infection with some of the

\footnotetext{
* Correspondence: Imermel@lifespan.org

${ }^{2}$ Department of Medicine, Warren Alpert Medical School of Brown University, Brown, USA

${ }^{3}$ Division of Infectious Diseases, Rhode Island Hospital, Rhode Island 593 Eddy St., Providence RI 02903, USA

Full list of author information is available at the end of the article
}

currently marketed antimicrobial CVCs [2-4]. However, there are no publications that have systematically reviewed prospective, randomized clinical trials comparing antimicrobial CVCs with non-antimicrobial CVCs to determine if there are differences in the incidence of species-specific CVC colonization that might suggest a lack of efficacy for specific pathogens. We hypothesized that some currently marketed antimicrobial CVCs may lack activity against specific microbial pathogens, and as such, may select for colonization and eventual bloodstream infection caused by such pathogens. We analyzed available data to determine if there is a proven benefit at the species-specific level for use of antimicrobial CVCs and to determine if some of these CVCs may have any vulnerability in their antimicrobial spectra. We studied CVC colonization rather than CVC-related bloodstream
C Biomed Central

(C) 2012 Novikov et al.; licensee BioMed Central Ltd. This is an Open Access article distributed under the terms of the Creative Commons Attribution License (http://creativecommons.org/licenses/by/2.0), which permits unrestricted use, distribution, and reproduction in any medium, provided the original work is properly cited. 
infection since endemic CVC infections start with microbial colonization prior to the development of CVC-related bloodstream infection. Additionally, most studies have shown a 2 to 10 -fold increase in episodes of CVC colonization compared to CVC-related bloodstream infection [2]. Thus, the greatest likelihood of uncovering any potential gaps in the spectrum of activity of antimicrobial CVCs would best be done by assessing CVC colonization.

\section{Materials and methods}

We reviewed prospective, randomized clinical trials comparing antimicrobial CVCs to non-antimicrobial control CVCs. Inclusion criteria were as previously described [2]. In brief, we performed a search in Cochrane, MEDLINE, and EMBASE databases of randomized controlled trials from 1995 to current with the following strings: "central venous catheter", "colonization", "catheter colonization", "bloodstream infection", "bacteremia", "chlorhexidine", "benzalkonium chloride", "rifampicin", "minocycline", "silver", and "miconazole". We chose to search from 1995 onwards as this was when the first study was published in those included in our first meta-analysis [2]. Catheter colonization was defined as at least 15 CFUs of microbial growth by semi-quantitative culture [5], or at least 1000 CFUs after quantitative vortex culture [6], or at least 100 CFUs after vortexing and sonication [6]. All statistical analyses were performed with MetAnalysis 1.0 software. Gart odds ratios (ORs) with 95\% CIs were calculated for each study that met entry criteria. For each antimicrobial CVC, we analyzed the number of antimicrobial CVCs colonized with a specific microorganism or related group of microorganisms as a fraction of all colonized antimicrobial CVCs of the same type. This fraction was compared with non-

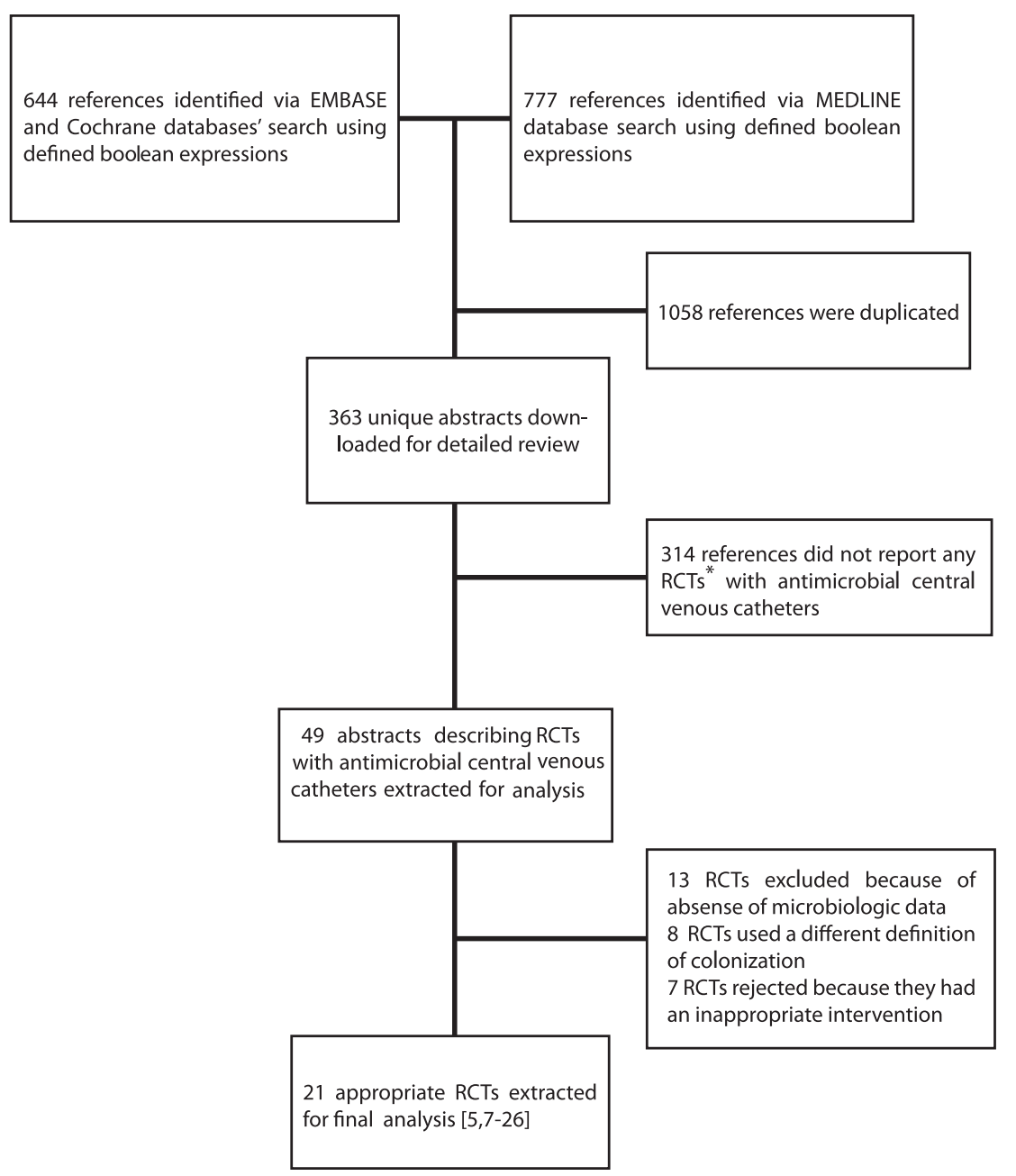

Figure 1 *RCT: Randomized controlled trial. 
antimicrobial CVCs used in the same studies analyzed in the same way.

\section{Microorganism colonized control CVCs All colonized control CVCs \\ vS. \\ Microorganism colonized test CVCs All colonized test $\mathrm{CVCs}$}

The rate of CVC colonization for each microorganism was analyzed separately and various pooled odds ratios (ORs) were calculated by both the Gart fixed-effects models (FEMs) and the DerSimonian-Laird random-effects models (REMs). The Cochran Q statistics and $\mathrm{I}^{2}$ test were used to assess heterogeneity. $\mathrm{I}^{2}$ values of $0 \%$ indicates no observed heterogeneity, whereas larger values indicated increasing heterogeneity. Results of the Gart FEM are quoted unless substantial heterogeneity was found, in which case the results of the DerSimonian-Laird REM are stated. We only included trials meeting our previously published criteria. Additionally, we only included those studies in which species-specific rates of colonization were delineated or those studies in which we were able to contact the authors directly to obtain species-specific colonization rates for antimicrobial and non-antimicrobial CVC groups. Our final analysis included an assessment for rates of CVC colonization for specific microbial species if available. If authors used the terms 'coliforms' or 'diphtheroids', we analyzed colonization rates but we did not combine these results with species-specific data. If the information regarding CVC colonization was insufficient, we attempted to contact the study corresponding author. We requested additional information from eleven authors; three authors responded before the final analysis was completed. As such, these latter studies [7-9] were included in the final analysis.

\section{Results}

Twenty-one randomized, controlled trials met our inclusion criteria [5,7-26] (Figure 1; Tables 1 and 2). Combining all antimicrobial CVCs, there was no significant difference in the proportion of colonized antimicrobial CVCs that were colonized by Staphylococcus aureus or coagulasenegative staphylococci compared to the corresponding

Table 1 Baseline characteristics of the studies used in the meta-analysis

\begin{tabular}{|c|c|c|c|c|c|c|}
\hline Study & CVC type & $\begin{array}{l}\text { Study } \\
\text { double-blinded? }\end{array}$ & $\begin{array}{l}\text { Guidewire } \\
\text { exchange } \\
\text { permitted? }\end{array}$ & $\begin{array}{l}\text { More than one } \\
\text { study CVC permitted } \\
\text { per patient? }\end{array}$ & $\begin{array}{l}\text { Proportion of } \\
\text { withdrawals or } \\
\text { dropouts }\end{array}$ & $\begin{array}{l}\text { Intention to } \\
\text { treat analysis } \\
\text { reported? }\end{array}$ \\
\hline Goldschmidt et al. 1995 [10] & Silver vs standard & - & no & yes & $12.4 \%$ & no \\
\hline Bach et al. 1996 [11] & CHSS vs. standard & - & no & no & - & no \\
\hline Ciresi et al. 1996 [12] & CHSS vs. standard & - & yes & yes & $5.4 \%$ & no \\
\hline van Heerden et al. 1996 [13] & CHSS vs. standard & - & no & no & $11.5 \%$ & no \\
\hline Maki et al. 1997 [5] & CHSS vs. standard & yes & yes & yes & $8.8 \%$ & no \\
\hline Heard et al. 1998 [7] & CHSS vs. standard & - & yes & yes & $15.6 \%$ & no \\
\hline Bach et al 1999 [14] & Silver vs standard & - & yes & no & $13 \%$ & no \\
\hline Collin et al. 1999 [15] & CHSS vs. standard & - & yes & yes & $2.1 \%$ & no \\
\hline Hannan et al. 1999 [16] & CHSS vs. standard & no & no & yes & - & no \\
\hline Marik et al. 1999 [17] & $\begin{array}{l}\text { CHSS vs. standard } \\
\text { and MR vs. standard }\end{array}$ & no & no & no & $5.8 \%$ & no \\
\hline Sheng et al. 2000 [18] & CHSS vs. standard & yes & no & yes & - & no \\
\hline Jaeger et al. 2001 [19] & $\begin{array}{l}\text { Benzalkonium chloride } \\
\text { vs. standard }\end{array}$ & no & no & no & - & no \\
\hline Corral et al. 2003 [20] & Silver vs standard & no & yes & yes & $19.8 \%$ & no \\
\hline Brun-Buisson et al. 2004 [21] & CHSS vs standard & yes & yes & yes & $8.6 \%$ & no \\
\hline Leon et al. 2004 [22] & MR vs. standard & no & no & no & $21.1 \%$ & yes \\
\hline Yucel et al. 2004 [23] & $\begin{array}{l}\text { Miconazole-rifampicin } \\
\text { vs. standard }\end{array}$ & no & no & no & $29.4 \%$ & no \\
\hline Dunser et al. 2005 [24] & Silver vs standard & no & no & no & - & no \\
\hline Rupp et al. 2005 [8] & CHSS vs. standard & yes & yes & no & $9.4 \%$ & yes \\
\hline Osma et al. 2006 [25] & CHSS vs. standard & - & no & no & $0 \%$ & yes \\
\hline Kalfon et al. 2007 [26] & Silver vs. standard & no & no & yes & $19.2 \%$ & no \\
\hline Raad et al.1997 [9] & MR vs. standard & no & no & yes & $10.7 \%$ & no \\
\hline
\end{tabular}

CVC (central venous catheter); CHSS (chlorhexidine - silver sulfadiazine); MR (minocycline-rifampin); "-" not reported. 
Table 2 Rates of colonization of the study and control catheters

\begin{tabular}{|c|c|c|c|c|}
\hline \multirow[t]{2}{*}{ Study } & \multirow{2}{*}{$\begin{array}{l}\text { Mean catheter dwell time } \\
\text { (test catheter days vs. } \\
\text { control catheter days) }\end{array}$} & \multirow{2}{*}{$\begin{array}{l}\text { Number of CVC } \\
\text { studied (test vs. } \\
\text { control) }\end{array}$} & \multicolumn{2}{|l|}{ Colonization } \\
\hline & & & $\mathrm{n}$ & Rate per 1000 days \\
\hline Goldschmidt et al. 1995 [10] & 13.3 vs 12.7 & 120 vs 113 & 54 (45.1\%) vs $50(44.2 \%)$ & 33.8 vs 34.8 \\
\hline Bach et al. 1996 [11] & 7.8 vs 7.8 & 116 vs 117 & $21(18.1 \%)$ vs $36(30.8 \%)$ & 23.2 vs 39.4 \\
\hline Ciresi et al. 1996 [12] & 12.9 vs 11.5 & 124 vs 127 & 10 (10.9\%) vs $12(12.1 \%)$ & 6.3 vs 8.2 \\
\hline van Heerden et al. 1996 [13] & 6.6 vs .6 .8 & 28 vs 26 & $4(14.3 \%)$ vs $10(38.5 \%)$ & 21.6 vs 56.6 \\
\hline Maki et al. 1997 [5] & 6 vs 6 & 208 vs 195 & 28 (13.5\%) vs 47 (24.1\%) & 22.4 vs 40.2 \\
\hline Heard et al. 1998 [7] & 8.5 vs 9.0 & 151 vs 157 & 60 (39.7\%) vs 81 (51.6\%) & 46.7 vs 57.3 \\
\hline Bach et al 1999 [14] & 4.5 vs 2.3 & 34 vs 33 & 9 (26.5\%) vs 7 (21.2\%) & 58.8 vs 52.2 \\
\hline Collin et al. 1999 [15] & 9.0 vs 7.3 & 98 vs 139 & $2(2.0 \%)$ vs 25 (18\%) & 2.3 vs 24.6 \\
\hline Hannan et al. 1999 [16] & 7.5 vs 7.6 & 174 vs 177 & 47 (27.2\%) vs $71(40.2 \%)$ & 36.0 vs 52.8 \\
\hline Marik et al. 1999 [17] & 6 vs 6 vs 6 & 36 vs 38 vs 39 & 7 (19.4\%) vs 4 (10.5\%) vs 11 (28.2\%) & 32.4 vs 17.5 vs 47.0 \\
\hline Sheng et al. 2000 [18] & 9.1 vs 8.2 & 113 vs 122 & $9(7.1 \%)$ vs $25(20.5 \%)$ & 8.8 vs 25 \\
\hline Jaeger et al. 2001 [19] & 14.8 vs 19.3 & 25 vs 25 & $4(16.0 \%)$ vs $4(16.0 \%)$ & 10.8 vs 8.3 \\
\hline Corral et al. 2003 [20] & 12 vs14 & 103 vs 103 & 29 (28.2\%) vs 41 (39.8\%) & 23.5 vs 27.7 \\
\hline Brun-Buisson et al. 2004 [21] & 10.5 vs 12.0 & 188 vs 175 & 7 (3.7\%) vs $23(13.1 \%)$ & 3.6 vs 11.0 \\
\hline Leon et al. 2004 [22] & 10.3 vs 10.4 & 187 vs 180 & $20(10.7 \%)$ vs $45(25.0 \%)$ & 10.4 vs 24.0 \\
\hline Yucel et al. 2004 [23] & 7.5 vs 6.7 & 118 vs 105 & $6(5.1 \%)$ vs $38(36.2 \%)$ & 6.8 vs 54.0 \\
\hline Dunser et al. 2005 [24] & 9.3 vs 9.7 vs 10.7 & 160 vs 165 vs 160 & $27(16.9 \%)$ vs 12 (7.3\%) vs 19 (11.9\%) & 18.1 vs 7.5 vs 11.9 \\
\hline Rupp et al. 2005 [8] & 6.9 vs. 6.7 & 384 vs 393 & $32(9.3 \%)$ vs $59(16.3 \%)$ & 12.1 vs 22.4 \\
\hline Osma et al. 2006 [25] & 11.7 vs 8.9 & 64 vs 69 & $14(21.9 \%)$ vs $14(20.3 \%)$ & 18.7 vs 22.8 \\
\hline Kalfon et al. 2007 [26] & 13.1 vs 12.9 & 320 vs 297 & $47(14.7 \%)$ vs $36(12.1 \%)$ & 11.2 vs 9.4 \\
\hline Raad et al. 1997 [9] & 6 vs 6 & 130 vs 136 & $11(8.5 \%)$ vs $36(26.5 \%)$ & 14.1 vs 44.1 \\
\hline
\end{tabular}

fractions of colonized control CVCs (Figures 2 and 3). Similarly, the proportion of $S$. aureus or coagulasenegative staphylococci colonized chlorhexidine-silver sulfadiazine, minocycline-rifampin, or silver antimicrobial CVCs was similar to the total proportion of colonized control CVCs. The proportion of miconazole-rifampin CVCs colonized with coagulase-negative staphylococci was less than the standard CVCs. However, this reflected the results of a single study [23].

The findings also demonstrated that there was no significant difference in the proportion of antimicrobial CVCs specifically colonized with Acinetobacter species compared to the proportion of Acinetobacter colonized control CVCs (Figure 4). Among those antimicrobial CVCs, the chlorhexidine-silver sulfadiazine CVCs colonized with Acinetobacter species were significantly less frequent, compared to corresponding colonized control CVCs (OR 0.16 [95\%CI 0.04-0.64]). However, publication bias was detected $(\mathrm{P}=0.01)$.

The proportion of antimicrobial CVCs that were colonized with diphtheroids was less than that of colonized control CVCs (OR 0.45 [95\%CI 0.25-0.79]). The proportion of colonized chlorhexidine-silver sulfadiazine CVCs that were colonized by diphtheroids was less than that of colonized standard control CVCs (OR 0.43 [95\%CI 0.23-0.82]).
Combining all antimicrobial CVCs, the proportion of colonized CVCs that were colonized by coliforms was greater than that of standard CVCs (OR 2.38 [95\%CI 1.105.15]). The proportion of coliform-colonized silver CVCs and minocycline-rifampin CVCs was greater than that of standard control CVCs (Figure 5). However, these findings each represented only a single clinical trial [20,22].

Combining all antimicrobial CVCs, there was no significant difference in the proportion of colonized antimicrobial CVCs colonized with Candida species compared to colonized standard CVCs (Figure 6). However, the proportion of colonized minocycline-rifampin CVCs colonized with Candida species was greater than that of colonized standard CVCs (OR 13.6 [95\%CI 4.2-43.4]).

\section{Discussion}

Antimicrobial-coated CVCs were developed in an effort to mitigate risk of serious CVC-related infections. Metaanalyses have shown that chlorhexidine-silver sulfadiazine CVCs, and particularly minocycline-rifampin CVCs, reduce the risk of CRBSI in prospective, randomized trials [2-4]. We compared data regarding colonization of antimicrobial and standard CVCs with known microorganisms that can cause CRBSI with an aim to investigate any potential vulnerability in the spectrum of antimicrobial activity. We 
found that chlorhexidine-silver sulfadiazine CVCs may have unique activity in reducing risk of colonization by Acinetobacter species and diphtheroids, but the former finding needs to be confirmed since we detected publication bias. Single study findings suggest that the miconazole-rifampin CVC may reduce the risk of colonization by coagulase- negative staphylococci, while the silver CVC and minocycline-rifampin CVC may be more vulnerable to coliform colonization, but these observations need to be confirmed by future studies. We identified a significant increase in the proportion of Candida species colonization among colonized minocycline-rifampin CVCs. Published 
clinical trials have not found an increased incidence of CVCrelated bloodstream infections due to Candida species but these studies are underpowered to detect such a difference.

Our findings regarding minocycline-rifampin CVC colonization with Candida species, as well as coliforms, support the observations of other investigators [22,27-29]. Some investigators found significantly less microbial adherence of Enterobacter aerogenes, Escherichia coli, Klebsiella pneumoniae, and C. albicans to chlorhexidine-silver sulfadiazine CVCs compared to non-antimicrobial CVCs but no such difference when minocycline-rifampin CVCs or silver CVCs were tested [28]. Additionally, they found increased microbial adherence of $C$. albicans to minocycline-rifampin CVCs compared to control CVCs in an in vitro model.

Our study has important limitations. We looked at CVC colonization rather than CVC-related bloodstream infection. However, as previously stated, for endemic intravascular CVC infections, CVC colonization is a prerequisite for bloodstream infection. As such, we feel that our findings have clinical relevance. We were unable to assess differences in CVC colonization based on the anatomic site of CVC insertion as this information was unavailable in the majority of the studies included in our analysis. Another potential weakness was the variable 


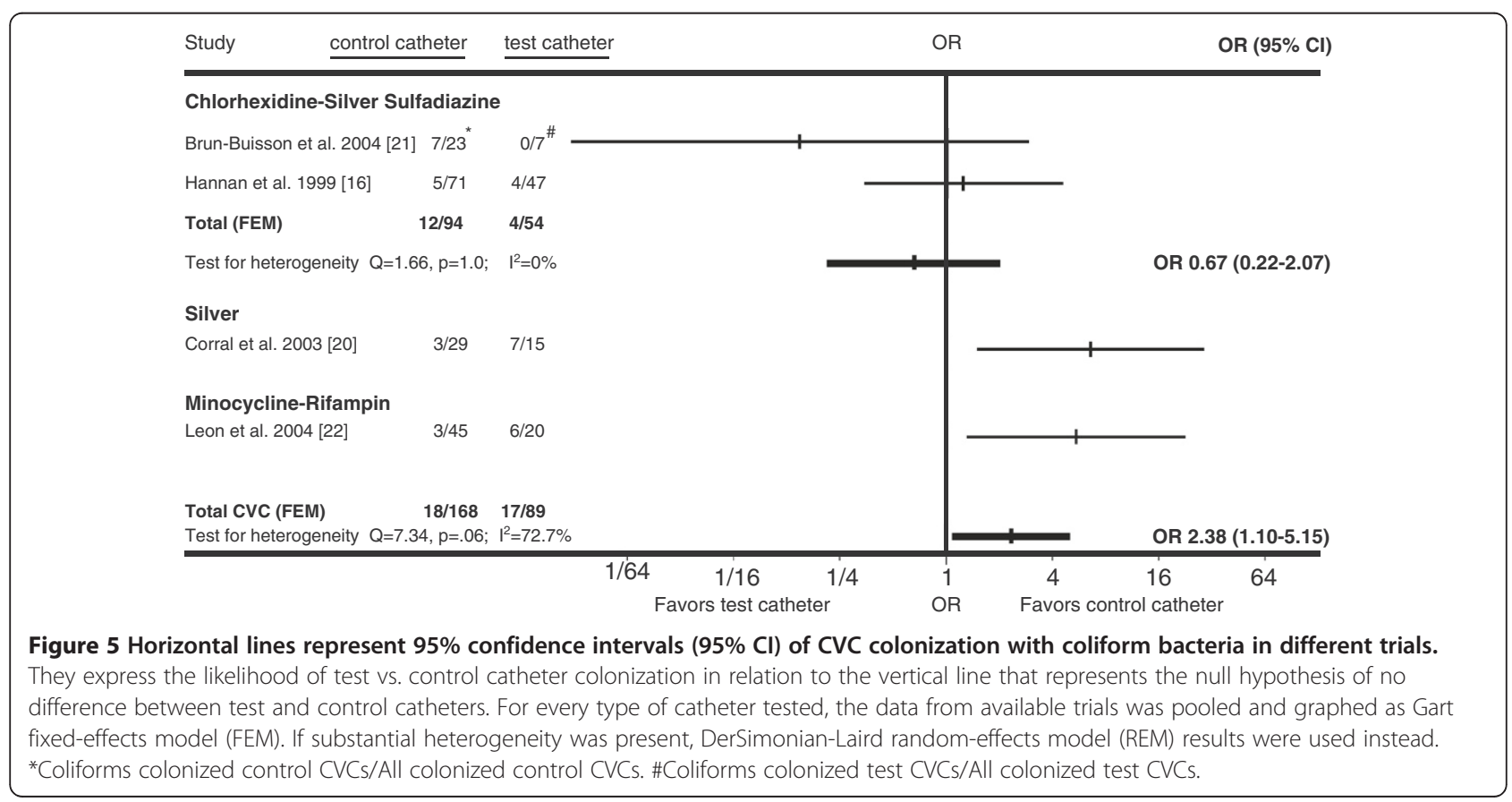


reported use of neutralizers when cultures of antimicrobial CVCs were performed.

Prospective studies and the ensuing meta-analyses have demonstrated the attributes of antimicrobial CVCs. However, we were interested in further understanding potential unintended consequences of widespread use of such devices. The data presented herein suggest that some antimicrobial CVCs may not reduce risk of CVC infections due to Candida or coliforms compared to uncoated CVCs despite showing overall benefit in clinical trials. As such, clinicians should weigh potential risks and benefits when contemplating use of specific antimicrobial CVCs in patients with prior colonization or infection due to these pathogens or when the patients are located in clinical areas where these microorganisms are endemic. Novel CVCs combining components of previously studied catheters have been demonstrated to have broader antimicrobial coverage and may well be less prone to colonization with select microorganisms as demonstrated in our investigation [30].

\section{Competing interests}

Dr. Mermel has had research funding from Astrellas and he has served as a consultant for Angiotech, Bard, Catheter Connections, Fresenius, ICU Medical, Semprus, and Teleflex. Dr. Elliott has received research funding from Carefusion, $3 \mathrm{M}$ and Becton Dickinson and has served on an advisory board for $3 \mathrm{M}$. Drs. Novikov, Lam, Casey and Nightingale have no competing interest. 


\section{Authors' contributions}

This project was initiated by LAM. AN, MYL, and ALC assisted in the metaanalysis; ALC and PN carried out statistical analysis; AN and MYL drafted the initial manuscript; ALC, TSE, PN, and LAM reviewed and amended the manuscript. All authors read and approved the final manuscript.

\section{Author details}

${ }^{1}$ Warren Alpert Medical School of Brown University, Brown, USA.

${ }^{2}$ Department of Medicine, Warren Alpert Medical School of Brown University, Brown, USA. ${ }^{3}$ Division of Infectious Diseases, Rhode Island Hospital, Rhode Island 593 Eddy St., Providence RI 02903, USA. ${ }^{4}$ Department of Clinical Microbiology, University Hospitals Birmingham NHS Foundation Trust, Birmingham, UK. ${ }^{5}$ Wolfson Computer Laboratory, University Hospitals Birmingham NHS Foundation Trust, Birmingham, UK.

Received: 23 July 2012 Accepted: 16 November 2012

Published: 3 December 2012

\section{References}

1. Mermel LA: What is the predominant source of intravascular catheter infections? Clin Infect Dis 2011, 52:211-212.

2. Casey AL, Mermel LA, Nightingale P, Elliott TSJ: Antimicrobial central venous catheters in adults: a systematic review and meta-analysis. Lancet Infect Dis 2008, 8:763-776.

3. Hockenhull JC, Dwan K, Boland A, Smith G, Bagust A, Dündar Y, Gamble C, McLeod C, Walley T, Dickson R: The clinical effectiveness and costeffectiveness of central venous catheters treated with anti-infective agents in preventing bloodstream infections: a systematic review and economic evaluation. Health Technol Assess 2008, 12:1-154.

4. Wang $H$, Huang $T$, Jing J, Jin J, Wang P, Yang M, Cui W, Zheng $Y$, Shen $H$ : Effectiveness of different central venous catheters for catheter-related infections: a network meta-analysis. J Hosp Infect 2010, 76:1-11.

5. Maki DG, Stolz SM, Wheeler S, Mermel LA: Prevention of central venous catheter-related bloodstream infection by use of an antisepticimpregnated catheter. A randomized, controlled trial. Ann Intern Med 1997, 127:257-266.

6. Cleri DJ, Corrado ML, Seligman SJ: Quantitative culture of intravenous catheters and other intravascular inserts. J Infect Dis 1980, 141:781-786.

7. Heard SO, Wagle M, Vijayakumar E, McLean S, Brueggemann A, Napolitano LM, Edwards LP, O'Connell FM, Puyana JC, Doern GV: Influence of triple-lumen central venous catheters coated with chlorhexidine and silver sulfadiazine on the incidence of catheter-related bacteremia. Arch Intern Med 1998, 158:81-87.

8. Rupp ME, Lisco SJ, Lipsett PA, Perl TM, Keating K, Civetta JM, Mermel LA, Lee D, Dellinger EP, Donahoe M, Giles D, Pfaller MA, Maki DG, Sherertz R: Effect of a second-generation venous catheter impregnated with chlorhexidine and silver sulfadiazine on central catheter-related infections: a randomized, controlled trial. Ann Intern Med 2005, 143:570-580.

9. Raad I, Darouiche R, Dupuis J, Abi-Said D, Gabrielli A, Hachem R, Wall M, Harris R, Jones J, Buzaid A, Robertson C, Shenaq S, Curling P, Burke T, Ericsson C: Central venous catheters coated with minocycline and rifampin for the prevention of catheter-related colonization and bloodstream infections. A randomized, double-blind trial. The Texas Medical Center Catheter Study Group. Ann Intern Med 1997, 127:267-274.

10. Goldschmidt H, Hahn U, Salwender HJ, Haas R, Jansen B, Wolbring P, Rinck $M$, Hunstein W: Prevention of catheter-related infections by silver coated central venous catheters in oncological patients. Zentralbl. Bakteriol. 1995, 283:215-223.

11. Bach AA, Schmidt HH, Böttiger BB, Schreiber BB, Böhrer HH, Motsch JJ, Martin EE, Sonntag HGH: Retention of antibacterial activity and bacterial colonization of antiseptic-bonded central venous catheters. J Antimicrob Chemother 1996, 37:315-322.

12. Ciresi DL, Albrecht RM, Volkers PA, Scholten DJ: Failure of antiseptic bonding to prevent central venous catheter-related infection and sepsis. Am Surg 1996, 62:641-646.

13. van Heerden PV, Webb SA, Fong S, Golledge CL, Roberts BL, Thompson WR: Central venous catheters revisited-infection rates and an assessment of the new Fibrin Analysing System brush. Anaesth Intensive Care 1996, 24:330-333.

14. Bach A, Eberhardt H, Frick A, Schmidt H, Böttiger BW, Martin E: Efficacy of silver-coating central venous catheters in reducing bacterial colonization. Crit Care Med 1999, 27:515-521.
15. Collin GR: Decreasing catheter colonization through the use of an antiseptic-impregnated catheter: a continuous quality improvement project. Chest 1999, 115:1632-1640.

16. Hannan M, Juste RN, Umasanker S, Glendenning A, Nightingale C, Azadian B, Soni N: Antiseptic-bonded central venous catheters and bacterial colonisation. Anaesthesia 1999, 54:868-872.

17. Marik PE, Abraham G, Careau P, Varon J, Fromm RE: The ex vivo antimicrobial activity and colonization rate of two antimicrobial-bonded central venous catheters. Crit Care Med 1999, 27:1128-1131.

18. Sheng WH, Ko WJ, Wang JT, Chang SC, Hsueh PR, Luh KT: Evaluation of antiseptic-impregnated central venous catheters for prevention of catheter-related infection in intensive care unit patients. Diagn Microbiol Infect Dis 2000, 38:1-5.

19. Jaeger K, Osthaus A, Heine J, Ruschulte H, Kuhlmann C, Weissbrodt H, Ganser A, Karthaus M: Efficacy of a benzalkonium chloride-impregnated central venous catheter to prevent catheter-associated infection in cancer patients. Chemotherapy 2001, 47:50-55.

20. Corral L, Nolla-Salas M, Ibañez-Nolla J, León MA, Díaz RM, Cruz Martín M, Iglesia R, Catalan R: A prospective, randomized study in critically ill patients using the Oligon Vantex catheter. J Hosp Infect 2003, 55:212-219.

21. Brun-Buisson C, Doyon F, Sollet J-P, Cochard J-F, Cohen Y, Nitenberg G: Prevention of intravascular catheter-related infection with newer chlorhexidine-silver sulfadiazine-coated catheters: a randomized controlled trial. Intensive Care Med 2004, 30:837-843.

22. León C, Ruiz-Santana S, Rello J, La Torre De MV, Vallés J, Alvarez-Lerma F, Sierra R, Saavedra P, Alvarez-Salgado F: Cabaña Study Group: benefits of minocycline and rifampin-impregnated central venous catheters. A prospective, randomized, double-blind, controlled, multicenter trial. Intensive Care Med 2004, 30:1891-1899.

23. Yücel N, Lefering R, Maegele M, Max M, Rossaint R, Koch A, Schwarz R, Korenkov M, Beuth J, Bach A, Schierholz J, Pulverer G, Neugebauer EAM: Reduced colonization and infection with miconazole-rifampicin modified central venous catheters: a randomized controlled clinical trial. $J$ Antimicrob Chemother 2004, 54:1 109-1115.

24. Dünser MW, Mayr AJ, Hinterberger G, Flörl CL, Ulmer H, Schmid S, Friesenecker B, Lorenz I, Hasibeder WR: Central venous catheter colonization in critically ill patients: a prospective, randomized, controlled study comparing standard with two antiseptic-impregnated catheters. Anesth Analg 2005, 101:1778-1784.

25. Osma S, Kahveci SF, Kaya FN, Akalin H, Ozakin C, Yilmaz E, Kutlay O: Efficacy of antiseptic-impregnated catheters on catheter colonization and catheter-related bloodstream infections in patients in an intensive care unit. J Hosp Infect 2006, 62:156-162.

26. Kalfon P, de Vaumas C, Samba D, Boulet E, Lefrant J-Y, Eyraud D, Lherm T, Santoli F, Naija W, Riou B: Comparison of silver-impregnated with standard multi-lumen central venous catheters in critically ill patients. Crit Care Med 2007, 35:1032-1039

27. Sampath LA, Tambe SM, Modak SM: In vitro and in vivo efficacy of catheters impregnated with antiseptics or antibiotics: evaluation of the risk of bacterial resistance to the antimicrobials in the catheters. Infect Control Hosp Epidemiol 2001, 22:640-646.

28. Gaonkar TA, Modak SM: Comparison of microbial adherence to antiseptic and antibiotic central venous catheters using a novel agar subcutaneous infection model. J Antimicrob Chemother 2003, 52:389-396.

29. Yorganci K, Krepel C, Weigelt JA, Edmiston CE: Activity of antibacterial impregnated central venous catheters against Klebsiella pneumoniae. Intensive Care Med 2002, 28:438-442.

30. Raad I, Mohamed JA, Reitzel RA, Jiang Y, Raad S, Al Shuaibi M, Chaftari AM, Hachem RY: Improved antibiotic-impregnated catheters with extended- spectrum activity against resistant bacteria and fungi. Antimicrob Agents Chemother 2012, 56:935-941.

doi:10.1186/2047-2994-1-40

Cite this article as: Novikov et al: Impact of catheter antimicrobial coating on species-specific risk of catheter colonization: a meta-analysis. Antimicrobial Resistance and Infection Control 2012 1:40. 